\title{
Topographic Organization of Hippocampal Inputs to the Anterior Olfactory Nucleus
}

\author{
Afif J. Aqrabawi ${ }^{1}$ and Jun Chul Kim ${ }^{1,2 *}$ \\ ${ }^{1}$ Department of Cell \& Systems Biology, University of Toronto, Toronto, ON, Canada, ${ }^{2}$ Department of Psychology, University \\ of Toronto, Toronto, ON, Canada
}

Top-down processes conveying contextual information play a major role in shaping odor representations within the olfactory system, yet the underlying mechanisms are poorly understood. The hippocampus (HPC) is a major source of olfactory top-down modulation, providing direct excitatory inputs to the anterior olfactory nucleus (AON). However, HPC-AON projections remain uncharacterized. In an effort to understand how hippocampal inputs are distributed within the AON, we systematically outlined their organization using anterograde and retrograde tracing methods. We found that AON-projecting hippocampal pyramidal neurons are located mostly in the ventral two-thirds of the HPC and are organized topographically such that cells with a ventral to intermediate hippocampal point of origin terminate, respectively, at the medial to lateral AON. Our neuroanatomical findings suggest a potential role for the HPC in the early processing and contextualization of odors which merits further investigation.

Keywords: AON, tracing, topography, hippocampus, olfaction, anterograde, retrograde, CA1

\section{INTRODUCTION}

OPEN ACCESS

Edited by:

Ricardo Insausti, Universidad de Castilla-La Mancha,

Spain

Reviewed by:

Joachim H. R. Lübke, Forschungszentrum Jülich, Germany Aldis Patrick Weible, University of Oregon, United States

${ }^{*}$ Correspondence: Jun Chul Kim

kim@psych.utoronto.ca

Received: 22 May 2017 Accepted: 07 February 2018 Published: 22 February 2018

Citation:

Aqrabawi AJ and Kim JC (2018) Topographic Organization of Hippocampal Inputs to the Anterior Olfactory Nucleus.

Front. Neuroanat. 12:12. doi: 10.3389/fnana.2018.00012
Olfaction is the primary sensory modality employed to perceive chemical signals in the environment. Within the olfactory bulb (OB), neural input arriving from olfactory sensory neurons terminate in glomeruli onto the dendrites of mitral and tufted cells (Vassar et al., 1994; Shepherd et al., 2004). The axons of these neurons fasciculate and form the lateral olfactory tract, a fiber system which courses the ventrolateral aspect of the brain and innervates the olfactory and entorhinal cortex, among other regions (Haberly and Price, 1977; Nagayama et al., 2010). Each olfactory sensory neuron expresses only one type of odorant receptor (out of $\sim 1000$ ) and all cells which express a common receptor type terminate their axons within a unique glomerulus. This allows olfactory stimuli to be represented in a pattern of activity elicited at the glomeruli termed an "odor map" (Ressler et al., 1994; Mombaerts et al., 1996). The neural representation is then processed further downstream where it is ultimately reconstructed into a perceived "odor object" (Wilson and Sullivan, 2011).

Although olfactory perception is largely shaped by bottom-up inputs received by the OB, it is also modulated by top-down inputs based upon prediction, expectation and memory of previous experiences (Freeman and Schneider, 1982; Kay and Laurent, 1999; Doucette and Restrepo, 2008; Mandairon and Linster, 2009; Moreno et al., 2009). Thus, the neural and behavioral responses to a given odorant can vary over time for an individual and also among members of the same species. Such top-down control of olfaction is thought to occur by means of strong and diverse feedback connections arriving from higher cortical and limbic structures (Fletcher and Chen, 2010). The hippocampus (HPC), via its direct excitatory inputs to the olfactory system, is thought to be a major source of top-down modulation (Martin et al., 2007; Aqrabawi et al., 2016). However, the underlying neural mechanism remains obscure. 
Central to understanding how the HPC influences olfaction is an appreciation of how hippocampal signals are organized within the olfactory system. Bulk labeling studies whereby axons of pyramidal cells are visualized with neuronal tracers have outlined a connection between the HPC and the olfactory cortex (Swanson and Cowan, 1977; de Olmos et al., 1978; Haberly and Price, 1978; Van Groen and Wyss, 1990; Cenquizca and Swanson, 2007). These studies revealed that hippocampal innervation of the olfactory system is most abundant in the anterior olfactory nucleus (AON), a ring-like cortical structure situated immediately posterior to the $\mathrm{OB}$ and anterior to the piriform cortex (Brunjes et al., 2005). However, these early efforts were limited in the scope of their investigation, addressing only the existence of the HPC-AON pathway. The present study is aimed at offering detailed insight into the anatomical connectivity between the HPC and the AON to better understand how hippocampal inputs influence olfaction.

In a series of anterograde and retrograde tracing experiments, we systematically examined the connectivity between the dorsoventral extent of the HPC and the medial, dorsal, and lateral aspects of the $\mathrm{AON}(\mathrm{mAON}, \mathrm{dAON}$ and $1 \mathrm{AON}$ respectively). Our results demonstrate a topographic gradient in the organization of hippocampal outputs to the AON, such that a ventral-to-intermediate point of origin in the CA1/Subiculum resulted in a medial-to-lateral point of termination at the AON.

\section{MATERIALS AND METHODS}

\section{Animals}

Forty-four 8-12 week old adult male C57Bl/6 mice (Charles River Laboratories, Quebec, QC, USA) were used. Prior to surgery, mice were group housed in a temperature-controlled room on a $12 \mathrm{~h}$ light/dark cycle with ad libitum access to food and water. All procedures were performed in accordance with the guidelines of the Canadian Council on Animal Care (CCAC) and the University of Toronto Animal Care Committee. Surgical procedures were performed on mice under general anesthesia with isoflurane and mounted onto a stereotaxic frame. The atlas of the mouse brain by Paxinos and Franklin (2007) was used to determine coordinates for guiding the stereotaxic infusion of tracers.

\section{Stereotaxic Surgery}

For anterograde tracing experiments, AAV2/9-hSynhChR2(H134R)-eYFP $\left(\sim 10^{12} \mathrm{GC} / \mathrm{ml}\right)$ purchased from the

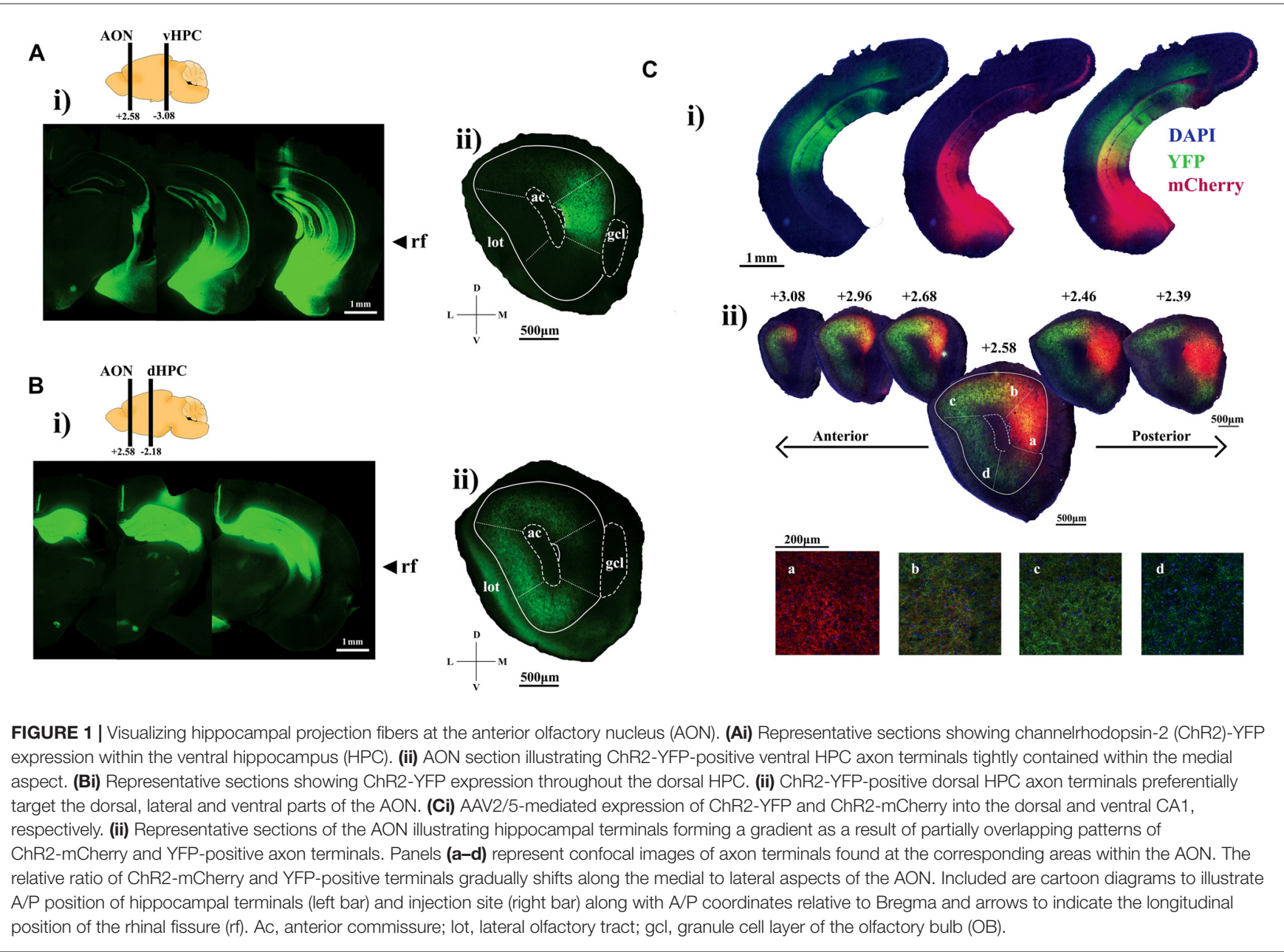


A
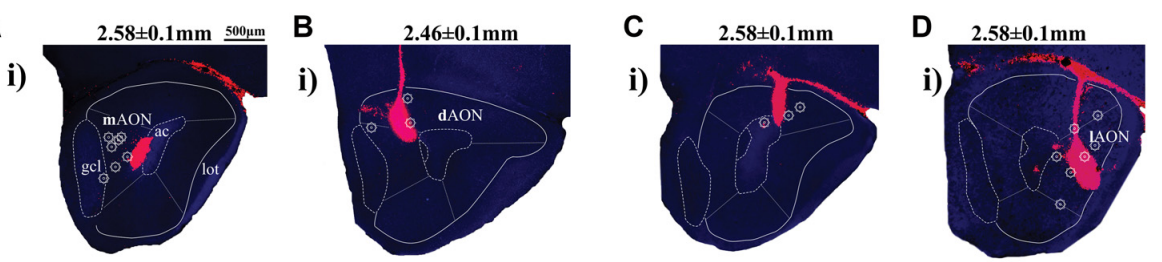

E

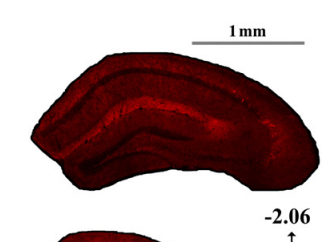

$\varliminf_{54}$
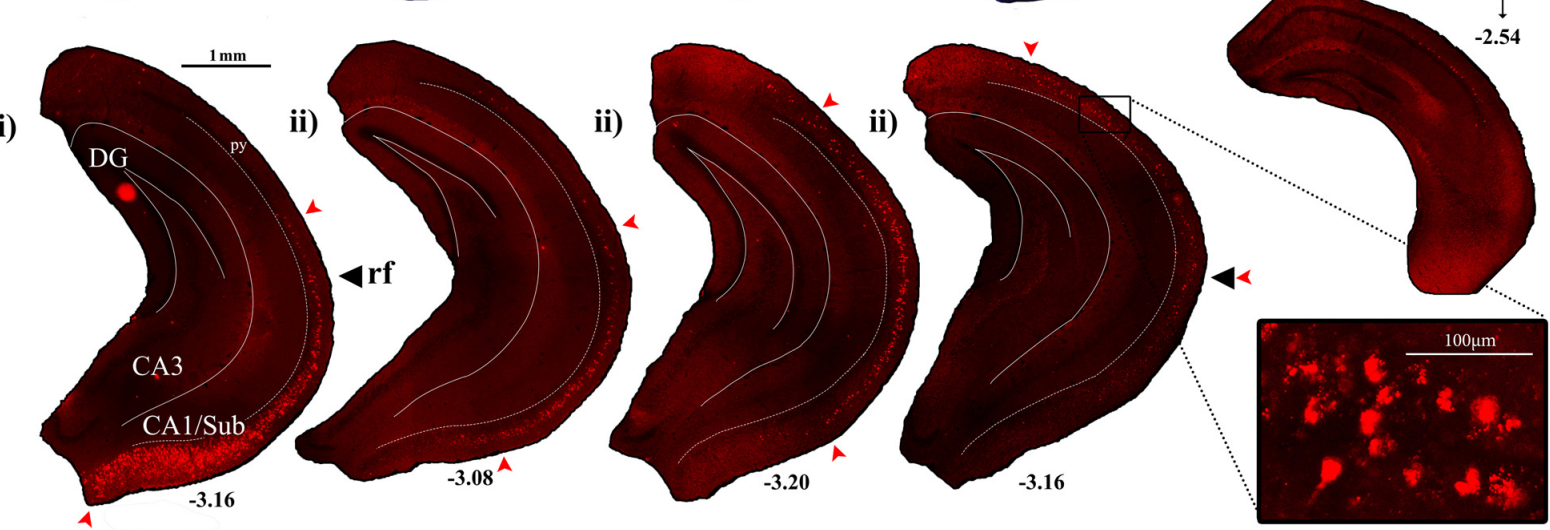

FIGURE 2 | Labeling patterns observed in the CA1 following injections of red fluorescent retrobeads into the AON. (Ai-Di) Representative sections depicting retrobead injection sites at the AON and the resulting cell body labeling patterns within the HPC. Included are AP coordinates to indicate position of coronal sections relative to Bregma and arrows to indicate the longitudinal position of the rf. The superimposed symbol (\$) indicates central positions of the injection sites which have yielded similar labeling patterns. (Aii) Retrobead injections restricted to the medial aspect of the AON produced labeled cell bodies found in the ventral-most CA1/Subiculum, decreasing in a gradient which ends at the level of the rf. (Bii) Injections delivered into the dorsomedial region of the AON resulted in labeled cell bodies found at their greatest density along more dorsal positions within the intermediate CA1. Few labeled cells were observed in the ventral-most CA1/Subiculum. (Cii) Injections into the dorsal AON resulted in retrobead-labeled cell bodies at the level of the rf, decreasing in number towards both dorsal and ventral directions. (Dii) Injections which targeted further lateral positions within the AON resulted in labeled cell bodies found at their greatest density in the intermediate CA1, decreasing in numbers towards the ventral CA1. The boxed region depicts a confocal image of retrobead-labeled pyramidal cells captured using a $20 \times$ objective. (E) Dorsal hippocampal coronal sections indicate the range of AP positions relative to Bregma where cell bodies were first identified following injections into the lateral $\mathrm{AON}$.

Vector Core at the University of Pennsylvania and a total of 18 mice were used. Two groups of six mice received unilateral infusions of $0.3 \mu \mathrm{L}$ of channelrhodopsin-2 (ChR2) into the dorsal HPC (no angle; two sets: AP: $-2.18 \mathrm{ML}: \pm 2.10 \mathrm{DV}$ : $-1.75, n=3$ or AP: $-2.70 \mathrm{ML}: \pm 2.20 \mathrm{DV}:-2.00, n=3$ ) or ventral $\mathrm{HPC}\left(10^{\circ}\right.$ angle away from midline; AP: $-2.92 \mathrm{ML}$ : $\pm 2.15 \mathrm{DV}:-4.90, n=6$ ). One group of six mice received a simultaneous co-injection of AAV2/5-hSyn-hChR2-mCherry $\left(\sim 10^{12} \mathrm{GC} / \mathrm{ml}\right)$ and AAV2/5-hSyn-hChR2-eYFP into the dorsal (AP: $-2.70 \mathrm{ML}: \pm 2.20 \mathrm{DV}:-2.00)$ and ventral HPC using a counterbalanced combination.

For the retrograde labeling experiments, a total of twenty four mice were used. Three groups of eight mice received a unilateral injection of $0.2 \mu \mathrm{L}$ of red fluorescent retrobeads (LumaFluor Inc., Naples, FL, USA) into the $\mathrm{mAON}\left(10^{\circ}\right.$ angle toward midline; AP: $+2.90 \mathrm{ML}: \pm 1.10 \mathrm{DV}:-3.42)$, dAON (no angle; two sets: AP: +2.80 ML: \pm 0.75 DV: $-3.10, n=4$ or AP: $+2.80 \mathrm{ML}: \pm 1.5 \mathrm{DV}:-3.10, n=4)$, or lAON (no angle; AP: +3.20 ML: \pm 1.10 DV: -3.90 ), respectively. A lower injection volume was used compared to anterograde tracing experiments to limit spread of the infusion outside the boundaries of the $\mathrm{AON}$ and neighboring AON subregions. All infusions were made by means of pressure ejection at a rate of $0.1 \mu \mathrm{L} / \mathrm{min}$ through a cannula connected by Tygon tubing to a $10 \mu \mathrm{L}$ Hamilton syringe (Hamilton, Reno, NV, USA) mounted onto an infusion pump. Following each infusion, the cannula was left in place for an additional $20 \mathrm{~min}$ before retraction to limit the spread of the tracer.

\section{Histology}

Approximately 3 weeks following surgery mice were transcardially perfused with Phosphate-Buffered Saline (PBS, $\mathrm{pH}$ 7.4), followed by $4 \%$ paraformaldehyde in phosphate buffer. Brains were extracted and postfixed overnight at $4^{\circ} \mathrm{C}$ and subsequently cryoprotected with PBS containing 30\% sucrose. Coronal $40 \mu \mathrm{m}$ thick sections were obtained using a cryostat (Leica, Germany). The sections were slide-mounted, counterstained with 4',6-diamidino-2-phenylindole (DAPI) for $10 \mathrm{~min}$, and coverslipped with Aquamount (Polysciences Inc., Warrington, PA, USA). The wide-field fluorescent images were captured with a $4 \times$ objective on a fluorescent microscope (Olympus, Japan). eYFP and red fluorescent retrobeads signals were captured using an U-MWIBA3 filter cube (Ex460- 495, Em510-550, DM505) and an U-MWIG3 filter cube (Ex530-550, Em575IF, DM570), respectively. Confocal images were captured through a Quorum spinning disk confocal microscope (Zeiss) using a $20 \times$ objective lens and 
were subsequently analyzed with Volocity Software (Perkin Elmer). eYFP and red fluorescent retrobeads signals were excited with 491 and $561 \mathrm{~nm}$ laser, respectively. Adobe Photoshop CS6 (Adobe Systems Incorporated, San Jose, CA, USA) was used to adjust the brightness and contrast of representative sections.

\section{Cell Counting}

Retrobead-positive cell counting was performed using the cellSens software (Olympus, Japan). The HPC was divided into three portions such that the dorsal one-third consisted of the anterior portion of the HPC prior to its transition into its longitudinal form in coronal sections (approximately $2.46 \mathrm{~mm}$ posterior to Bregma). The remaining ventral two-third of the HPC was further divided into intermediate and ventral HPC separated by the rhinal fissure (rf). Calibration parameters were established using randomly chosen tissue sections and were selected such that the threshold for cell diameter was high enough to exclude the majority of falsely identified cells (noise). Calibration parameters were maintained for consistent counting of all tissue sections. Tissue sections from three animals in each of the medial, dorsal, and lateral-AON injected groups were analyzed. The density of retrobead-positive cells found across nine coronal sections (number of labeled cells in region of interest/area of region) for each subject, and mean densities for each group were calculated.

\section{RESULTS}

\section{ChR2-YFP-mediated Anterograde Tracing Reveals the Topographic Organization of HPC Inputs to the AON}

To examine the hippocampal innervation pattern of the AON, we first performed antero- grade tracing of hippocampal projections using virally delivered-ChR2 as an anterograde tracer (Gradinaru et al., 2009; Jennings and Stuber, 2015). AAV-hSyn-ChR2-YFP (ChR2 fused to eYFP driven by the human synapsin promoter) was infused into either the ventral (Figure 1Ai) or dorsal HPC (Figure 1Bi). Viral infusions targeting the ventral HPC led to expression of ChR2-YFP in the ventral half of the HPC below the rf, while dorsal HPC infusions led to ChR2-YFP expression in the dorsal half of the HPC above the rf (Figures 1Ai,Bi). In both cases, ChR2-YFP expression was restricted to the HPC with minimal spread into adjacent cortical structures such as the primary somatosensory cortex which does not project to the AON (Zakiewicz et al., 2014).

ChR2-YFP-positive axon fibers originating from the ventral half of the HPC were localized largely at the mAON with little signal observed in the $\mathrm{OB}$, piriform cortex, or other AON subregions (Figure 1Aii). In contrast, projections from the dorsal half of the HPC were found predominantly in the dorsal, lateral, and ventral, but not medial, aspects of the AON, indicating a topographic organization of HPC inputs within the AON (Figure 1Bii). We confirmed this differential innervation pattern using two-color anterograde tracing by simultaneously expressing ChR2-YFP and ChR2-mCherry in the dorsal and ventral halves of the HPC, respectively (Figure 1Ci). Hippocampal projections established a gradient of innervation within the AON whereby YFP-positive and mCherry-positive HPC axon fibers partially overlapped at the junction between the dorsal and medial AON. The density of projections originating from the ventral half of the HPC gradually increased from the lateral to the medial $\mathrm{AON}$ whereas projections from the dorsal half of the HPC increased gradually from the medial to lateral AON (Figure 1Cii).

\section{Topographic Organization of HPC Inputs to the AON Is Further Confirmed by Retrograde Tracing}

The ChR2-mediated anterograde tracing method cannot unambiguously distinguish hippocampal axon terminals from hippocampal fibers of passage at the AON. Furthermore, it remains unclear how the AON-projecting $\mathrm{HPC}$ cells are distributed along the dorso-ventral axis of the HPC. To overcome this limitation and further validate the topographic organization of hippocampal inputs, we performed retrograde tracing experiments by employing fluorescent retrogradelytransported microspheres (retrobeads) that are uptaken at presynaptic axon terminals. Retrobeads were microinjected unilaterally into the medial, dorsal, or lateral aspects of the AON in separate cohorts of mice ( $n=4-8$ for each group). The spread of injected retrobeads was restricted to the targeted AON subregions (Figures 2Ai-Di), which are denominated on the basis of their cardinal positions as previously described (Brunjes et al., 2005). In all cases, the majority of labeled cells

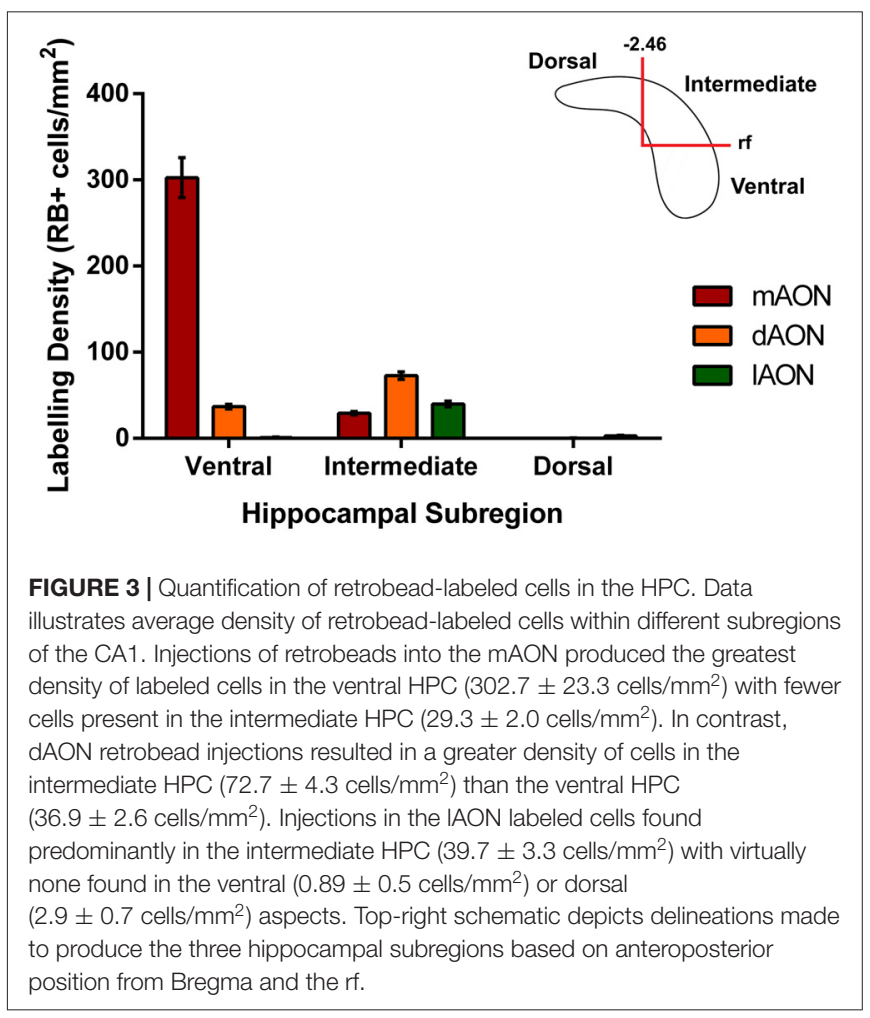



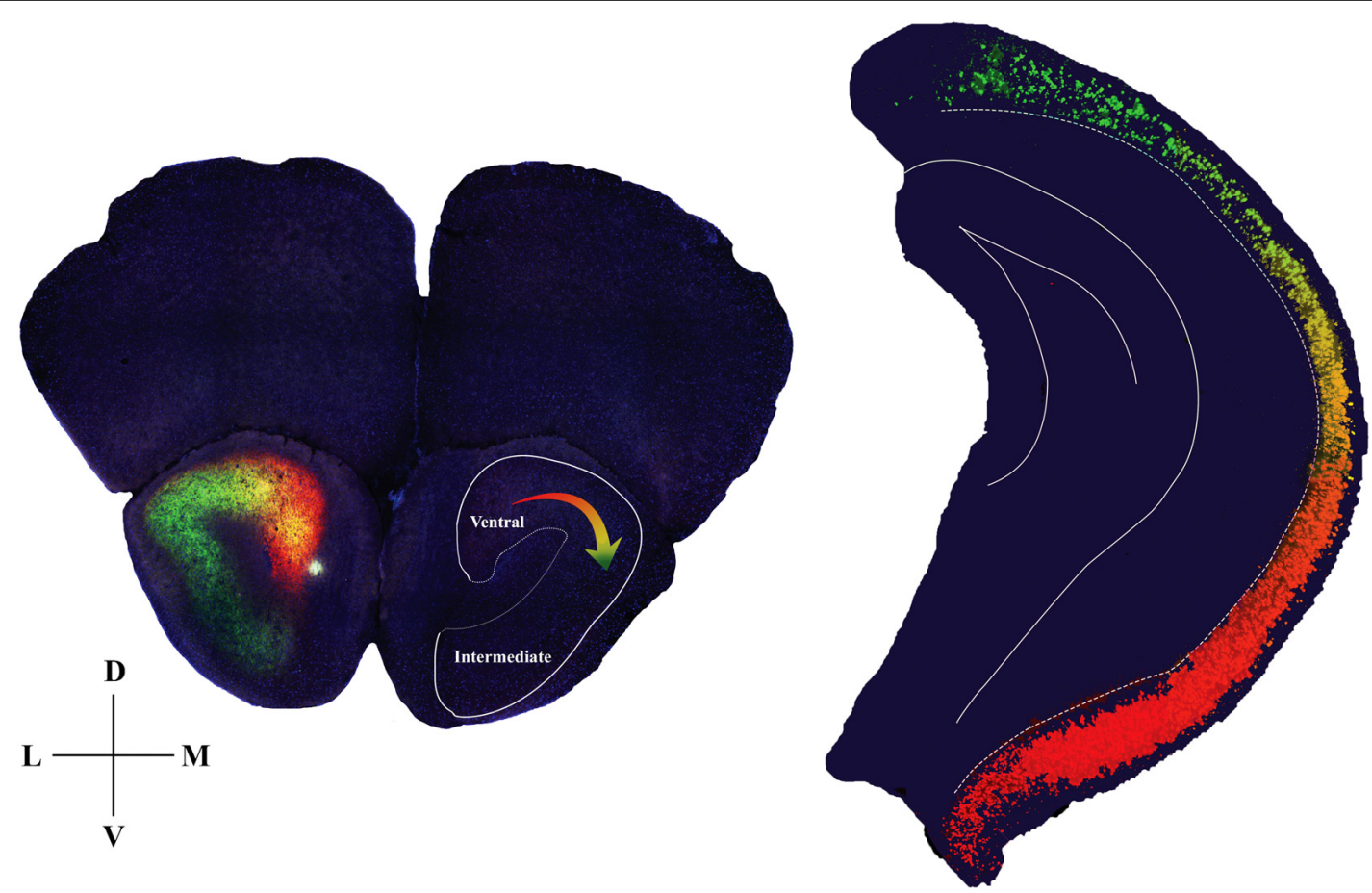

FIGURE 4 | Diagram summarizing anatomical details of the hippocampal-AON pathway. Coronal section of the AON depicting CA1 pyramidal cell terminals in a gradient of innervation (left) which reflects the position of the cells (pseudo-colored from retrobead signals) within the hippocampal longitudinal axis (right). In the opposite hemisphere an outline is drawn to represent a hippocampal image that is created as a result of this innervation pattern.

were observed in the hemisphere ipsilateral to the injection site, with very few cells labeled in the contralateral HPC (data not shown).

Injection of retrobeads into the mAON yielded labeled cells found in their greatest density near the ventral-most part of the CA1/Subiculum subregion (Figures 2Aii, 3). These $\mathrm{mAON}$-projecting cells in the ventral CA1 progressively decreased in number towards the dorsal end of the longitudinal axis, forming a gradient terminating at the level of the rf. In contrast, retrobead injections into the $\mathrm{dAON}$ produced the greatest density of labeled cells in the intermediate CA1 at or near the level of the rf, tapering gradually in both dorsal and ventral directions (Figures 2Bii,Cii, 3). Very few, if any, dAON-projecting cells were found at the ventral- and dorsal-most parts of the CA1. Finally, $1 \mathrm{AON}$ injections produced retrobead-labeled cells which were found in their greatest density at the dorsal aspect of the intermediate CA1 and were progressively fewer towards the ventral end with the gradient terminating at the level of the rf (Figures 2Dii, 3). Notably, 1AON injections yielded extremely sparse, if any, labeling in the dorsal one-third of the CA1 (Figure 2E); instead, labeled cells first appeared at the dorsal most extent of the ventral two-thirds of the CA1 (Bregma: $\sim-2.06$ to -2.54 ; Figure 2E), indicating that the dorsal CA1 is unlikely to represent a meaningful source of inputs to the AON. Taken together, our anterograde and retrograde tracing data revealed that hippocampal inputs originating from the ventral two-third of the HPC are topographically organized within the AON.

\section{DISCUSSION}

The present study has revealed a topographic gradient in HPC-AON projections such that the ventral-most part of the HPC innervates most heavily the mAON, and progressively more dorsal parts of the HPC innervate increasingly more lateral positions at the AON as illustrated in our model (Figure 4). This pattern of connectivity suggests that the ventral two-third of the HPC superimposes an image of its entire dorsoventral axis onto the AON. This would also imply that the AON can be mapped in detail to reflect activity in an associated hippocampal area, in a manner analogous to the cortical homunculus representing a layout of the body.

Our tracing results are largely consistent with previous studies which examined the downstream targets of CA1 projection neurons (Swanson and Cowan, 1977; de Olmos et al., 1978; Haberly and Price, 1978; Van Groen and Wyss, 1990; Cenquizca and Swanson, 2007). AON-projecting hippocampal fibers join the white matter of the alveus and course through the fimbriafornix to reach the lateral septum, nucleus accumbens, and the prefrontal cortex before they innervate the AON (Cenquizca and Swanson, 2007; Arszovszki et al., 2014). Alternatively, hippocampal fibers may also reach the AON via the longitudinal association bundle through which the HPC extends its axons to other sensory and visceral cortical areas. Our tracing methods do not distinguish these two routes, thus it remains to be determined to what extent each route contributes to the formation of the topographic innervation pattern observed at the AON. 
The functional connection between the HPC and the olfactory system is growing increasingly apparent. The HPC has been implicated in different aspects of odor processing, including olfactory pattern separation, the formation of context-odor associations, coding of the temporal sequence of a series of odors, and olfactory learning and memory (Eichenbaum, 1998; Levy et al., 2004; Kent et al., 2007; Kesner et al., 2010, 2011; Weeden et al., 2014). Our anterograde tracing data demonstrate that hippocampal innervation of the olfactory system is largely restricted to the AON, suggesting that the AON plays a major role in mediating hippocampal modulation of olfaction. Further support for this idea is the AON's optimal position as the initial recipient of input from the $\mathrm{OB}$ and the largest source of olfactory cortical feedback projections (Carson, 1984). The AON also maintains extensive connections to other cortical regions such as the entorhinal cortex, amygdala, hypothalamus and neuromodulatory centers, though little is known beyond its anatomical and electrophysiological properties (Haberly and Price, 1978; Brunjes et al., 2005).

The functional implication of the gradient in the HPC-AON connection remains unclear. Notably, a similar topographic organization is known to exist in other extrinsic targets of the HPC including the amygdala, nucleus accumbens, and lateral septum (Groenewegen et al., 1987; Risold and Swanson, 1997; Witter et al., 2000; Kishi et al., 2006). The HPC is well-documented to play a role in a variety of cognitive and emotional operations, among them are learning, memory, spatial navigation, anxiety and stress responseassociated processing (for a review see Strange et al., 2014). Of note, a study by Dong et al. (2009) showed that the CA1 can be divided into three subregions based on the expression pattern of molecular spatial markers: dorsal (dCA1), intermediate (iCA1), and ventral (vCA1) CA1. The study found that the dCA1 occupies approximately dorsal one-third of the CA1 whereas iCA1 and vCA1 together occupy the ventral two-third of the CA1 (Dong et al., 2009) thereby providing a more precise organization scheme as an alternative to the

\section{REFERENCES}

Aikath, D., Aldis, P. W., Rowland, D. C., and Kentros, C. G. (2014). Role of self-generated odor cues in contextual representation. Hippocampus 24, 1039-1051. doi: 10.1002/hipo.22289

Aqrabawi, A. J., Browne, C. J., Dargaei, Z., Garand, D., Khademullah, C. S., Woodin, M. A., et al. (2016). Top-down modulation of olfactory-guided behaviours by the anterior olfactory nucleus pars medialis and ventral hippocampus. Nat. Commun. 7:13721. doi: 10.1038/ncomms13721

Arszovszki, A., Borhegyi, Z., and Klausberger, T. (2014). Three axonal projection routes of individual pyramidal cells in the ventral CA1 hippocampus. Front. Neuroanat. 8:53. doi: 10.3389/fnana.2014.00053

Brunjes, P. C., Illig, K. R., and Meyer, E. A. (2005). A field guide to the anterior olfactory nucleus (cortex). Brain Res. Rev. 50, 305-335. doi: 10.1016/j. brainresrev.2005.08.005

Carson, K. A. (1984). Quantitative localization of neurons projecting to the mouse main olfactory bulb. Brain Res. Bull. 6, 629-634. doi: 10.1016/03619230(84)90143-6

Cenquizca, L. A., and Swanson, L. W. (2007). Spatial organization of direct hippocampal field CA1 axonal projections to the rest of the cerebral cortex. Brain Res. Rev. 56, 1-26. doi: 10.1016/j.brainresrev.2007.05.002 often-arbitrary reference to dorsal and ventral HPC (Fanselow and Dong, 2010). Our results show that hippocampal inputs to the AON arise from the iCA1 and vCA1, but not dCA1, and that the iCA1 and vCA1 innervate the medial to lateral AON, respectively. The lack of feedback projections from the dCA 1 is consistent with studies suggesting that odor information processing is restricted to the ventral CA1, yet this view remains controversial owing to recent findings which implicate olfactory involvement in dorsal HPC function, particularly in place field development (Kulvicius et al., 2008; Aikath et al., 2014; Igarashi et al., 2014; Zhang and Manahan-Vaughan, 2015). Thus, the functional role of the HPC-AON pathway can only be clear following further investigations into both structures.

Ultimately, the topographic organization of the HPC-AON pathway offers a unique model for investigating how information originating from distinct hippocampal subregions contribute to olfaction. In an initial effort to elucidate the role of hippocampal inputs to the AON, we recently showed that the vCA1-mAON pathway can modulate olfactory sensitivity (Aqrabawi et al., 2016). However, considering the well-established role of HPC in representing spatiotemporal context, it would be of great interest to investigate how $\mathrm{iCA} 1$ and vCA1 inputs to the AON differentially regulate olfactory memory.

\section{AUTHOR CONTRIBUTIONS}

AJA and JCK carried out the study conceptualization and experimental design and wrote the manuscript. AJA performed the surgical procedures.

\section{FUNDING}

This research was funded by operating grants to JCK from the Canadian Institutes for Health Research (MOP 496401) and the Natural Sciences and Engineering Council of Canada (NSERC; MOP 491009).

de Olmos, J., Hardy, H., and Heimer, L. (1978). The afferent connections of the main and the accessory olfactory bulb formations in the rat: an experimental HRP-study. J. Comp. Neurol. 181, 213-244. doi: 10.1002/cne.901810202

Dong, H. W., Swanson, L. W., Chen, L., Fanselow, M. S., and Toga, A. W. (2009). Genomic-anatomic evidence for distinct functional domains in hippocampal field CA1. Proc. Natl. Acad. Sci. U S A 106, 11794-11799. doi: 10.1073/pnas. 0812608106

Doucette, W., and Restrepo, D. (2008). Profound context-dependent plasticity of mitral cell responses in olfactory bulb. PLoS Biol. 6:e258. doi: 10.1371/journal. pbio. 0060258

Eichenbaum, H. (1998). Using olfaction to study memory. Ann. N Y Acad. Sci. 855, 657-669. doi: 10.1111/j.1749-6632.1998.tb10642.x

Fanselow, M. S., and Dong, H. W. (2010). Are the dorsal and ventral hippocampus functionally distinct structures? Neuron 65, 7-19. doi: 10.1016/j.neuron.2009. 11.031

Fletcher, M. L., and Chen, W. R. (2010). Neural correlates of olfactory learning: critical role of centrifugal neuromodulation. Learn. Mem. 17, 561-570. doi: 10.1101/lm.941510

Freeman, W. J., and Schneider, W. (1982). Changes in spatial patterns of rabbit olfactory EEG with conditioning to odors. Psychophysiology 19, 44-56. doi: $10.1111 /$ j.1469-8986.1982.tb02598.x 
Gradinaru, V., Mogri, M., Thompson, K. R., Henderson, J. M., and Deisseroth, K. (2009). Optical deconstruction of parkinsonian neural circuitry. Science 324, 354-359. doi: 10.1126/science.1167093

Groenewegen, H. J., Vermeulen-Van der Zee, E., te Kortschot, A., and Witter, M. P. (1987). Organization of the projections from the subiculum to the ventral striatum in the rat. A study using anterograde transport of Phaseolus vulgaris leucoagglutinin. Neuroscience 23, 103-120. doi: 10.1016/03064522(87)90275-2

Haberly, L. B., and Price, J. L. (1977). The axonal projection patterns of the mitral and tufted cells of the olfactory bulb in the rat. Brain Res. 129, 152-157. doi: 10.1016/0006-8993(77)90978-7

Haberly, L. B., and Price, J. L. (1978). Association and commissural fiber systems of the olfactory cortex of the rat. II. systems originating in the olfactory peduncle. J. Comp. Neurol. 181, 781-807. doi: 10.1002/cne.901810407

Igarashi, K. M., Lu, L., Colgin, L. L., Moser, M. B., and Moser, E. I. (2014). Coordination of entorhinal-hippocampal ensemble activity during associative learning. Nature 510, 143-147. doi: 10.1038/nature13162

Jennings, J. H., and Stuber, G. D. (2015). Tools for resolving functional activity and connectivity within intact neural circuits. Curr. Biol. 24, R41-R50. doi: 10.1016/j.cub.2013.11.042

Kay, L. M., and Laurent, G. (1999). Odor- and context-dependent modulation of mitral cell activity in behaving rats. Nat. Neurosci. 2, 1003-1009. doi: $10.1038 / 14801$

Kent, K., Hess, K., Tonegawa, S., and Small, S. A. (2007). CA3 NMDA receptors are required for experience-dependent shifts in hippocampal activity. Hippocampus 17, 1003-1011. doi: 10.1002/hipo.20332

Kesner, R. P., Hunsaker, M. R., and Ziegler, W. (2010). The role of the dorsal and ventral CA1 in memory for the temporal order of a sequence of odors. Neurobiol. Learn. Mem. 93, 111-116. doi: 10.1016/j.nlm.2009.08.010

Kesner, R. P., Hunsaker, M. R., and Ziegler, W. (2011). The role of the dorsal and ventral hippocampus in olfactory working memory. Neurobiol. Learn. Mem. 96, 361-366. doi: 10.1016/j.nlm.2011.06.011

Kishi, T., Tsumori, T., Yokota, S., and Yasui, Y. (2006). Topographical projection from the hippocampal formation to the amygdala: a combined anterograde and retrograde tracing study in the rat. J. Comp. Neurol. 496, 349-368. doi: 10.1002/cne.20919

Kulvicius, T., Tamosiunaite, M., Ainge, J., Dudchenko, P., and Wörgötter, F. (2008). Odor supported place cell model and goal navigation in rodents. J. Comput. Neurosci. 25, 481-500. doi: 10.1007/s10827-008-0090-x

Levy, D. A., Hopkins, R. O., and Squire, L. R. (2004). Impaired odor recognition memory in patients with hippocampal lesions. Learn. Mem. 11, 794-796. doi: 10.1101/lm.82504

Mandairon, N., and Linster, C. (2009). Odor perception and olfactory bulb plasticity in adult mammals. J. Neurophysiol. 101, 2204-2209. doi: 10.1152/jn. 00076.2009

Martin, C., Beshel, J., and Kay, L. M. (2007). An olfacto-hippocampal network is dynamically involved in odor-discrimination learning. J. Neurophysiol. 98, 2196-2205. doi: 10.1152/jn.00524.2007

Mombaerts, P., Wang, F., Dulac, C., Chao, S. K., Nemes, A., Mendelsohn, M., et al. (1996). Visualizing an olfactory sensory map. Cell 87, 675-686. doi: 10.1016/s0092-8674(00)81387-2

Moreno, M. M., Linster, C., Escanilla, O., Sacquet, J., Didier, A., and Mandairon, N. (2009). Olfactory perceptual learning requires adult neurogenesis. Proc. Natl. Acad. Sci. U S A 106, 17980-17985. doi: 10.1073/pnas.0907063106
Nagayama, S., Enerva, A., Fletcher, M. L., Masurkar, A. V., Igarashi, K. M., Mori, K., et al. (2010). Differential axonal projection of mitral and tufted cells in the mouse main olfactory system. Front. Neural Circuits 4:120. doi: 10.3389/fncir.2010.00120

Paxinos, G., and Franklin, K. B. J. (2007). The Mouse Brain in Stereotaxic Coordinates. 3rd Edn. San Diego, CA: Academic Press.

Ressler, K. J., Sullivan, S. L., and Buck, L. B. (1994). Information coding in the olfactory system: evidence for a stereotyped and highly organized epitope map in the olfactory bulb. Cell 79, 1245-1255. doi: 10.1016/0092-8674(94) 90015-9

Risold, P., and Swanson, L. (1997). Connections of the rat lateral septal complex. Brain Res. Rev. 24, 115-195. doi: 10.1016/s0165-0173(97)00009-x

Shepherd, G. M., Chen, W. R., and Greer, C. A. (2004). "Olfactory bulbeditor," in The Synaptic Organization of the Brain, ed. G. M. Shepherd (New York, NY: Oxford University Press), 159-204.

Strange, B. A., Witter, M. P., Lein, E. S., and Moser, E. I. (2014). Functional organization of the hippocampal longitudinal axis. Nat. Rev. Neurosci. 15, 655-669. doi: 10.1038/nrn3785

Swanson, L. W., and Cowan, W. M. (1977). An autoradiographic study of the organization of the efferent connections of the hippocampal formation in the rat. J. Comp. Neurol. 172, 49-84. doi: 10.1002/cne.901720104

Van Groen, T., and Wyss, J. M. (1990). Extrinsic projections from area CA1 of the rat hippocampus: olfactory, cortical, subcortical and bilateral hippocampal formation projections. J. Comp. Neurol. 302, 515-528. doi: 10.1002/cne. 903020308

Vassar, R., Chao, S. K., Sitcheran, R., Nunñz, J. M., Vosshall, L. B., and Axel, R. (1994). Topographic organization of sensory projection to the olfactory bulb. Cell 79, 981-991. doi: 10.1016/0092-8674(94)90029-9

Weeden, C. S., Hu, N. J., and Kesner, R. P. (2014). The role of the ventral dentate gyrus in olfactory pattern separation. Hippocampus 24, 553-559. doi: 10.1002/hipo.22248

Wilson, D. A., and Sullivan, R. M. (2011). Cortical processing of odour objects. Neuron 72, 506-519. doi: 10.1016/j.neuron.2011.10.027

Witter, M. P., Wouterlood, F. G., Naber, P. A., and Van Haeften, T. (2000). Anatomical organization of the parahippocampal-hippocampal network. Ann. N Y Acad. Sci. 911, 1-24. doi: 10.1111/j.1749-6632.2000.tb06716.x

Zakiewicz, I. M., Bjaalie, J. G., and Leergaard, T. B. (2014). Brain-wide map of efferent projections from rat barrel cortex. Front. Neuroinform. 8:5. doi: 10.3389/fninf.2014.00005

Zhang, S., and Manahan-Vaughan, D. (2015). Spatial olfactory learning contributes to place field formation in the hippocampus. Cereb. Cortex 25 , 423-432. doi: 10.1093/cercor/bht239

Conflict of Interest Statement: The authors declare that the research was conducted in the absence of any commercial or financial relationships that could be construed as a potential conflict of interest.

Copyright (C) 2018 Aqrabawi and Kim. This is an open-access article distributed under the terms of the Creative Commons Attribution License (CC BY). The use, distribution or reproduction in other forums is permitted, provided the original author(s) and the copyright owner are credited and that the original publication in this journal is cited, in accordance with accepted academic practice. No use, distribution or reproduction is permitted which does not comply with these terms. 